\title{
Machining Technology Optimization of Middle-convex and Varying Ellipse Piston Based on Fuzzy Logic
}

\author{
Yanwei XU ${ }^{1,2, a^{*}}$, Kai Zhang, ${ }^{2, b}$, Jun Dama ${ }^{2, c}$ and Tancheng $X^{2, d}$ \\ ${ }^{1}$ School of Mechanical Engineering, Tianjin University, Tianjin, 300072, China \\ ${ }^{2}$ School of Mechatronics Engineering, Henan University of Science and Technology, Luoyang, \\ 471003, China \\ axuyanweiluoyang@163.com, 'bhangkai@163.com, 'majunda@163.com, xietc@126.com \\ ${ }^{*}$ Corresponding author
}

\begin{abstract}
Keywords: Machining precision evaluation, Middle-convex and varying ellipse piston, Fuzzy logic Abstract. Machining precision of the middle-convex and varying ellipse piston is an important aspect influenced by machining technological, and influencing the functional performance of engine directly. The scientific and rational machining technological is the key to determine the manufacturing quality and cost-performance ratio of the middle-convex and varying ellipse piston. Machining technological evaluation of the middle-convex and varying ellipse piston is one important and complicated process influenced by many different factors directly, the fuzzy comprehensive evaluation method is proposed to evaluate the machining technological of the middle-convex and varying ellipse piston based on fuzzy logic. Finally, an example of machining technological evaluation on one certain middle-convex and varying ellipse piston for the illustration of the proposed methodology is presented. The feasibility and rationality of the methodology are verified by the experimental result.
\end{abstract}

\section{Introduction}

The middle-convex and varying ellipse piston is known as the heart of engine, its machining technological influences the functional performance of engine directly, and the scientific and rational machining technological also is the key to determine the manufacturing quality and cost-performance ratio of the middle-convex and varying ellipse piston [1-4]. However, how to evaluate the machining technological of middle-convex and varying ellipse piston synthetically is one complicated problem for the many different influencing factors.

The fuzzy comprehensive evaluation is one kind of effective multitudinous factors decision method used to evaluate the object synthetically affected by various factors [4-8], and the influence factors of the machining technological of middle-convex and varying ellipse piston always are uncertain [9-11], so the fuzzy comprehensive evaluation is the optimum method to evaluate machining technological of middle-convex and varying ellipse piston.

\section{Decision-making of machining technological of piston based on fuzzy logic}

\section{Establishment of influence factor set}

Influence factor set includes various factors influencing the machining technological design scheme directly. Manufacturing quality, machining cost and machining efficiency are the key considerations in decision-making of machining technological of middle-convex and varying ellipse piston design scheme. The influencing factor of manufacturing quality includes machining precision, surface roughness, dimensional precision and geometrical precision. The influencing factor of machining cost mainly takes into account wages, maintenance costs and depreciation expense of the manufacturing equipment, fixture costs and other expense. The influencing factor of machining efficiency mainly includes readiness time, installation and chucking time, machining time, auxiliary time and break time.

The influence factor set $U$ of decision-making of machining technological is expressed as 


$$
U=\left(\begin{array}{lll}
u_{1} & u_{2} & u_{3}
\end{array}\right)
$$

here, subset $u_{1}, u_{2}, u_{3}$ respectively indicates manufacturing quality, machining cost and machining efficiency.

The manufacturing quality subset $u_{1}$ is expressed as

$$
u_{1}=\left(\begin{array}{llll}
u_{11} & u_{12} & u_{13} & u_{14}
\end{array}\right)
$$

here, $u_{11}, u_{12}, u_{13}, u_{14}$ respectively indicates machining precision, surface roughness, dimensional precision and geometrical precision.

The machining cost subset $u_{2}$ is expressed as

$$
u_{2}=\left(\begin{array}{lllll}
u_{21} & u_{22} & u_{23} & u_{24} & u_{25}
\end{array}\right)
$$

here, $u_{21}, u_{22}, u_{23}, u_{24}, u_{25}$ respectively indicates wages, maintenance costs, depreciation expense, fixture costs and other expense.

The machining efficiency subset $u_{3}$ is expressed as

$$
u_{3}=\left(\begin{array}{lllll}
u_{31} & u_{32} & u_{33} & u_{34} & u_{35}
\end{array}\right)
$$

here, $u_{31}, u_{32}, u_{33}, u_{34}, u_{35}$ respectively indicates readiness time, installation and chucking time, machining time, auxiliary time and break time.

\section{Establishment of evaluation set}

Evaluation set is comprised of various possible evaluate results provided by the decision makers. Evaluation set of manufacturing quality subset $V_{1}$ is expressed as

$$
V_{1}=\left(\begin{array}{lllll}
\mathrm{A}_{1} & \mathrm{~B}_{1} & \mathrm{C}_{1} & \mathrm{D}_{1} & \mathrm{E}_{1}
\end{array}\right)
$$

here, $A_{1}, B_{1}, C_{1}, D_{1}, E_{1}$ respectively indicates good, relatively good, general, relatively bad and very bad.

Evaluation set of machining cost subset $V_{2}$ is expressed as

$$
V_{2}=\left(\begin{array}{lllll}
\mathrm{A}_{2} & \mathrm{~B}_{2} & \mathrm{C}_{2} & \mathrm{D}_{2} & \mathrm{E}_{2}
\end{array}\right)
$$

here, $A_{2}, B_{2}, C_{2}, D_{2}, E_{2}$ respectively indicates high, relatively high, general, low and very low.

Evaluation set of machining efficiency subset $V_{3}$ is expressed as

$$
V_{3}=\left(\begin{array}{lllll}
\mathrm{A}_{3} & \mathrm{~B}_{3} & \mathrm{C}_{3} & \mathrm{D}_{3} & \mathrm{E}_{3}
\end{array}\right)
$$

here, $A_{3}, B_{3}, C_{3}, D_{3}, E_{3}$ respectively indicates high, relatively high, general, low and very low.

To simplify problems, the evaluation sets of manufacturing quality subset $V_{1}$, machining cost subset $V_{2}$ and machining efficiency subset $V_{3}$, are unified into one evaluation set $V$, and it is expressed as

$$
V=\left(\begin{array}{lllll}
v_{1} & v_{2} & v_{3} & v_{4} & v_{5}
\end{array}\right)
$$


here, $v_{1}, v_{2}, v_{3}, v_{4}, v_{5}$ respectively indicates excellent, good, middle, pass muster and bad.

\section{Establishment of weight set}

Weight set is comprised of weight number reflecting the importance of every influence factor. Assuming $a_{i}$ is the weight number of influence factor $u_{i}$, weight set $A$ is expressed as

$$
A=\left(\begin{array}{llll}
a_{1} & a_{2} & \mathrm{~L} & a_{m}
\end{array}\right)
$$

Usually, the weight number of all influence factors need to satisfy the polarity and nonnegativity conditions. i.e.

$$
\left\{\begin{array}{l}
\sum_{i=1}^{m} a_{i}=1 \\
0 \leq a_{i} \leq 1
\end{array}\right.
$$

Toward one same evaluation target, different evaluator maybe has the different attitude, and the weight numbers offered by evaluators also are different. In this paper, the weighting method is adopted to determine the weight number of every influence factor. Firstly, to make a weight distribution questionnaire (shown as table 1), and then distributing the questionnaire to some experts and related people to fill in the optimum weight number who believe, finally, taking bake the weight distribution questionnaires and calculating the weight number $A$ of the evaluation target using weighting method.

Table 1. Weight Distribution Questionnaire

\begin{tabular}{llllll}
\hline influence factor & $u_{i}$ & $u_{1}$ & $u_{2}$ & $u_{3}$ & $\Sigma$ \\
\hline weight number & $a_{i}$ & $a_{1}$ & $a_{2}$ & $a_{3}$ & 1 \\
\hline
\end{tabular}

The weight set $A$ is calculated through the statistical investigation of the influence weight on decision-making of machining technological of the middle-convex and varying ellipse piston of the subsets $u_{1}, u_{2}$ and $u_{3}$. The result of statistical calculation is expressed as

$$
A=\left(\begin{array}{lll}
0.537 & 0.185 & 0.278
\end{array}\right)
$$

As the same way, the results of statistical calculation of the weight sets $A_{1}, A_{2}$ and $A_{3}$ of the subsets $u_{1}, u_{2}$ and $u_{3}$ also are expressed as

$$
\begin{aligned}
& A_{1}=\left(\begin{array}{lllll}
0.337 & 0.365 & 0.146 & 0.152
\end{array}\right) \\
& A_{2}=\left(\begin{array}{lllll}
0.255 & 0.217 & 0.216 & 0.159 & 0.153
\end{array}\right) \\
& A_{3}=\left(\begin{array}{lllll}
0.136 & 0.147 & 0.388 & 0.173 & 0.156
\end{array}\right)
\end{aligned}
$$

\section{Determination of the Membership Function}

Determine the degree of membership is one of the most important sectors to determine the degree of membership for decision-making on the machining technological of the middle-convex and varying ellipse piston using the methodology of fuzzy synthetic evaluation influencing the evaluate results 
directly. The degree of membership of the $\mathrm{j}$-th evaluating indicator of the influence factor $u_{i}$ can be calculated with the membership function. The weight of the evaluating indicator $a_{i j}$ or the span of the value $a_{i j}$ of the degree of membership $\mu_{i j}$ can be calculated using the methodology of fuzzy statistical analysis on the degree of membership of the $\mathrm{j}$-th evaluating indicator of the influence factor $u_{i}$ provided by every expert. The value $a_{i j}$ of the degree of membership of the influence factor $\mu_{i j}$ is shown as table 2 .

Table 2. The Value $a_{i j}$ of the Degree of Membership of $\mu_{i j}$

\begin{tabular}{cccccc}
\hline factor set & factor subset & $v_{1}$ & $v_{2}$ & $\mathbf{L}$ & $v_{m}$ \\
\hline & $u_{i 1}$ & $a_{10}-a_{11}$ & $a_{11}-a_{12}$ & $\mathbf{L}$ & $a_{1 m-1}-a_{1 m}$ \\
$u_{i}$ & $u_{i 2}$ & $a_{20}-a_{21}$ & $a_{21}-a_{22}$ & $\mathbf{L}$ & $a_{2 m-1}-a_{2 m}$ \\
& $\Gamma$ & $\Gamma$ & $\Gamma$ & $\Gamma$ & $\Gamma$ \\
& $u_{i n}$ & $a_{n 0}-a_{n 1}$ & $a_{n 1}-a_{n 2}$ & $\mathbf{L}$ & $a_{n m-1}-a_{n m}$ \\
\hline
\end{tabular}

Based on the table 2, the membership function is structured as follows

$$
\mu_{i 1}(x)=\left\{\begin{array}{cc}
1 & \left(a_{i 0} \leq x \leq a_{i 1}\right) \\
\left(a_{i 2}-x\right) /\left(a_{i 2}-a_{i 1}\right) & \left(a_{i 1} \leq x \leq a_{i 2}\right) \\
0 & a_{i 2} \leq x \leq a_{i m}
\end{array}\right.
$$

here, $i=1,2, \mathrm{~L}, n$.

$$
\mu_{i j}(x)= \begin{cases}0 & \left(a_{i 0} \leq x \leq a_{i j-2}\right) \\ \left(x-a_{i j-2}\right) /\left(a_{i j-1}-a_{i j-2}\right) & \left(a_{i j-2} \leq x \leq a_{i j-1}\right) \\ 1 & \left(a_{i j-1} \leq x \leq a_{i j}\right) \\ \left(a_{i j+1}-x\right) /\left(a_{i j+1}-a_{i j}\right) & \left(a_{i j+1} \leq x \leq a_{i m}\right) \\ 0 & \left(a_{i j} \leq x \leq a_{i j+1}\right)\end{cases}
$$

here, $i=1,2, \mathrm{~L}, n$ and $j=2,3, \mathrm{~L}, m-1$.

$$
\mu_{i m}(x)=\left\{\begin{array}{lc}
0 & \left(a_{i 0} \leq x \leq a_{i m-2}\right) \\
\left(x-a_{i m-2}\right) /\left(a_{i m-1}-a_{i m-2}\right) & \left(a_{i m-2} \leq x \leq a_{i m-1}\right) \\
1 & \left(a_{i m-1} \leq x \leq a_{i m}\right)
\end{array}\right.
$$

here, $i=1,2, \mathrm{~L}, n$.

Degree of membership $\mu_{i j}$ of the $j$-th evaluating indicator of the influence factor $u_{i}$ on one certain machining technological of the middle-convex and varying ellipse piston can be calculated through the membership function, equations (15), (16) and (17). 


\section{Fuzzy Comprehensive Evaluation}

\section{Primary fuzzy comprehensive evaluation}

Assuming the primary fuzzy comprehensive evaluation is evaluated on the influence factor $u_{i}$ in the influence set $U$, the degree of membership $\mu_{i j}$ of the $\mathrm{j}$-th evaluating indicator of the influence factor $u_{i}$ can be calculated through the membership function, and the evaluation result of the single factor $u_{i}$ can be expressed by the fuzzy set $R_{i j}$

$$
R_{i j}=\frac{\mu_{i 1}}{v_{1}}+\frac{\mu_{i 2}}{v_{2}}+\mathrm{L}+\frac{\mu_{i m}}{v_{n}}
$$

here, $R_{i j}$ is the evaluation set of the single factor, it is expressed simply as

$$
R_{i j}=\left(\begin{array}{llll}
r_{i 1} & r_{i 2} & \mathrm{~L} & r_{i m}
\end{array}\right)
$$

Similarly, the evaluation set corresponding to every influence factor $u_{i}$ can be obtained. And the single factor evaluation matrix $R_{i}$ is expressed as

$$
R_{i}=\left[\begin{array}{llll}
R_{i 1} & R_{i 2} & \mathrm{~L} & R_{i 4}
\end{array}\right]^{T}
$$

here, $R_{i}$ is the single factor fuzzy evaluation matrix.

The primary evaluation set $B_{i}$ can be obtained through the fuzzy comprehensive evaluation on the influence factor $u_{i}$.

$$
B_{i}=A_{i} \mathrm{O} R_{i}=\left(\begin{array}{llll}
b_{i 1} & b_{i 2} & \mathrm{~L} & b_{i m}
\end{array}\right)
$$

\section{Secondary fuzzy comprehensive evaluation}

With the primary evaluation set $B_{1}, B_{2}, \mathrm{~L}$, and $B_{k}$, the single factor evaluation matrix $R$ of the influence factor set $U$ can be expressed as

$$
R=\left[\begin{array}{c}
B_{1} \\
B_{2} \\
\mathrm{M} \\
B_{K}
\end{array}\right]=\left[\begin{array}{cccc}
b_{11} & b_{12} & \mathrm{~L} & b_{1 m} \\
b_{21} & b_{22} & \mathrm{~L} & b_{2 m} \\
\mathrm{M} & \mathrm{M} & \mathrm{M} & \mathrm{M} \\
b_{k 1} & b_{k 2} & \mathrm{~L} & b_{k m}
\end{array}\right]
$$

The fuzzy comprehensive evaluation set $B$ can be obtained through the fuzzy comprehensive evaluation on the influence factor set $U$, and it can be expressed as

$$
B=A O R=\left(\begin{array}{llll}
b_{1} & b_{2} & \mathrm{~L} & b_{m}
\end{array}\right)
$$

The fuzzy comprehensive evaluation on one certain machining technological of the middle-convex and varying ellipse piston can be carried out with the method of maximum degree of membership, the weighted average method or the fuzzy distribution method through the normalization of the fuzzy comprehensive evaluation set $B$. 


\section{Example of Machining Technology Evaluation of Middle-convex and Varying Ellipse Piston}

An example of machining technology evaluation of middle-convex and varying ellipse piston is illustrated. The diameter of workpiece is $\phi 70$, machining precision is IT7, roughness concentration is Ra1.6, ovality is 0.6 , upper deviation is $e_{s}=0.02$, lower deviation is $e_{i}=0$, material is aluminium casting alloy. It needs to select the optimum machining technological from the four alternatives. The degrees of membership of manufacturing quality, machining cost and machining efficiency of every machining technological design scheme are calculated (shown as table 3) with primary fuzzy comprehensive evaluation based on the membership function.

Table 3. Degree of Membership of Influence Factors

\begin{tabular}{cccc}
\hline alternative design scheme & manufacturing quality & machining cost & machining efficiency \\
\hline 1 & 0.422 & 0.277 & 0.309 \\
2 & 0.509 & 0.483 & 0.519 \\
3 & 0.553 & 0.516 & 0.563 \\
4 & 0.466 & 0.548 & 0.415 \\
\hline
\end{tabular}

The evaluation matrix $R$ of the single factor of the four alternatives is expressed as equation (24) based on the degrees of membership of manufacturing quality, machining cost and machining efficiency of every machining technological design scheme.

$$
R=\left[\begin{array}{llll}
0.422 & 0.509 & 0.553 & 0.466 \\
0.277 & 0.483 & 0.516 & 0.548 \\
0.309 & 0.519 & 0.563 & 0.415
\end{array}\right]
$$

The four alternatives and the decision-making value of every machining technological of the middle-convex and varying ellipse piston clculated with the method of maximum degree of membership are shown as table 4. Finally, the third machining technological is chosen as the optimum design scheme from the result of the fuzzy comprehensive evaluation of the four machining technological design schemes.

Table 4. Value of Fuzzy Comprehensive Evaluation

\begin{tabular}{|c|c|c|}
\hline number & machining technological design scheme & evaluation value \\
\hline 1 & a rough turning $\boldsymbol{b}$ diecapitated $\propto$ chamfering $\mathbb{e}$ radial facing & 0.422 \\
\hline 2 & a rough turning $\boldsymbol{b}$ half finish turning $\propto$ radial facing $\mathbb{d}$ grinding & 0.509 \\
\hline 3 & a rough turning $\Phi$ half finish turning $\propto$ radial facing $\propto$ finish turning & 0.537 \\
\hline 4 & $\propto$ rough turning $b$ half finish turning $\propto$ finish turning $\mathbb{e}$ chamfering & 0.466 \\
\hline
\end{tabular}

\section{Conclusion}

Evaluation of machining technological of the middle-convex and varying ellipse piston is a typical combinatorial optimization problem in manufacturing technologies. Fuzzy comprehensive evaluation is one effective evaluation methodology for multitudinous factors. In this paper, the evaluation methodology of the machining technological of the middle-convex and varying ellipse piston based on fuzzy comprehensive evaluation is proposed, and the optimum machining technological of the middle-convex and varying ellipse piston is chosen from the alternatives of one given work-piece using the methodology. It can avoid the rigidity of the decision model and correct some error of judgment in the traditional methodology of decision-making on machining technological of the middle-convex and varying ellipse piston, and it also can optimize the machining technological and improve the intelligece 
of decision-making on the machining technological design scheme of the middle-convex and varying ellipse piston.

\section{Acknowledgements}

The authors are grateful for the financial support of the National Natural Science Foundation of China (51305127) and the scientific research key project fund of the Education Department Henan Province of China (14A460018).

\section{References}

[1] Huang, Haibin; Guo, Yinbiao; Xie, Shutong; Jiang, Chen. Research on control technology of the middle-convex and varying ellipse piston lathe system [C]. 2009 IEEE International Conference on Intelligent Computing and Intelligent Systems, ICIS 2009, v 2, p 622-626.

[2] Wang, X.; Jiang, S.; Qian, L.; Shi, Z.; Wu, D. Research on the on-line measuring system for the middle-convex and varying ellipse piston skirt $[\mathrm{J}]$. Chinese Journal of Mechanical Engineering (English Edition), v 14, n 4, p 305-309, December 2001.

[3] Zheng, Dong ; Zhou, Hui Xing; Wu, Han; Zhang, Chao Yang. Development of non-contact online measuring system for middle-convex and varying ellipse piston [J]. Advanced Materials Research, v 753-755, p 2282-2285, 2013, Materials Processing and Manufacturing III.

[4] Xie, Tancheng; Zhang, Jianguo; Xin, Hongmin; Xu, Yanwei; Nan, Xiang. Research on CNC and Lathe processing of middle-convex and varying piston [C]. Advanced Materials Research, v 317-319, p 1832-1836, 2011, Equipment Manufacturing Technology and Automation.

[5] Luo, Jin-Yao; Qiu, Yuan-Feng. Fuzzy comprehensive evaluation for decision making of water saving irrigation system [J]. Wuhan University Journal of Natural Sciences, v 8, n 3 A, p 837-841, September 2003.

[6] Wang, Yan; Le, Jiajin; Huang, Dongmei. Research on the fuzzy comprehensive evaluation of performance analysis in the data warehouse engineering model design [C]. IMETI 2010 - 3rd International Multi-Conference on Engineering and Technological Innovation, Proceedings, v 1, p 35-39, 2010.

[7] Deng, Guohua. A application of fuzzy comprehensive evaluation in information systems supplier evaluation [C]. Proceedings - 2011 4th International Conference on Information Management, Innovation Management and Industrial Engineering, ICIII 2011, v 3, p 509-513, 2011.

[8] Zhang, Xiaohui; Wang, Lin; Yin, Qi. The assessment of the location of reverse logistics processing center based on fuzzy comprehensive evaluation [C]. Proceedings of the 2013 3rd International Conference on Intelligent System Design and Engineering Applications, ISDEA 2013, p 1400-1403.

[9] Cui, Hong; Cui, Jinghu. Study on anti-disturbance of linear servo system used for piston machining [J]. Applied Mechanics and Materials, v 29-32, p 1970-1975, 2010, Applied Mechanics and Mechanical Engineering.

[10] Xin, Hongmin; Shi, Yaoyao;Xie, Tancheng; Zhang, Jianguo; Sun, Pengcheng. A piston lathing technology using double-cutter frame feeding mechanism [J]. Xibei Gongye Daxue Xuebao/Journal of Northwestern Polytechnical University, v 32, n 3, p 457-463, June 2014.(Language: Chinese).

[11] Xie, Shu-Tong; Guo, Yin-Biao; Yang, Qing-Quan; Chen, Lu-Shuang. Research on the shaping approach for non-cylinder piston turning [C]. 2009 International Conference on Measuring Technology and Mechatronics Automation, ICMTMA 2009, v 3, p 129-132. 